\title{
Erratum
}

\section{Spatial Prediction and Ordinary Kriging}

Incorrect equations were given (Cressie, 1988) in the section describing the role of the range in ordinary kriging, which are corrected below. However, the general conclusion that the range is not very informative about the kriging neighborhood size remains unchanged.

The expression for kriging weights $l$ in Eq. (28) is incorrect. The expression should be

$$
\underset{\sim}{l}=\Sigma^{-1}\left(\underset{\sim}{\sigma}+\frac{\left(1-\underline{\sigma}^{\prime} \Sigma^{-1} \underset{\sim}{1}\right)}{{\underset{\sim}{\prime}}^{\prime} \Sigma^{-1} \underset{\sim}{1}}\right)
$$

Then, if $\underset{\sim}{\sigma}=\underset{\sim}{0}, \underset{\sim}{l}=\Sigma^{-1} 1 /\left(\underline{\sim}^{\prime} \Sigma^{-1} 1\right)$.

The subsequent development is unchanged, except that Eq. (30) should be (for $0<f \leqq 1 / 2$ )

$$
\hat{Z}\left(s_{0}\right)=\left[2 Z\left(s_{1}\right)+(3-f) Z\left(s_{2}\right)-Z\left(s_{3}\right)\right] /(4-f)
$$

And Eq. (31) should be (for $1 / 2<f \leqq 1$ )

$\hat{Z}\left(s_{0}\right)=\left[\left(5 f-2 f^{2}\right) Z\left(s_{1}\right)+(2+f) Z\left(s_{2}\right)-2(1-f) Z\left(s_{3}\right)\right] /\left(8 f-2 f^{2}\right)$

The conclusions made about these predictors remain the same.

Finally, on p. 419, for the example where $C(h)=\exp (-\lambda|h|) C(0), s_{i}=$ $i ; i=1, \ldots, n$, and $s_{0}=0, l$ should be $[\alpha+\mathrm{O}(1 / n), \mathrm{O}(1 / n), \ldots, \mathrm{O}(1 / n)]$. In other words, the optimal prediction of $Z\left(s_{0}\right)$ is approximately $\alpha$ times the mean-corrected nearest value, expressing the regression effect caused by the spatial correlation.

If the range does not provide guidance to the choice of a kriging neighborhood, what does? Rivoirard (1987) shows that a minimal neighborhood for 
which $\left(1-\sigma_{\tilde{\sigma}}^{\prime} \Sigma^{-1}{ }_{\sim}^{1}\right) /\left(1_{\sim}^{\prime} \Sigma^{-1} 1\right)$ is small, is a sensible criterion for choosing locations to be included in the ordinary kriging predictor $\hat{Z}\left(s_{0}\right)$.

Noel Cressie

Department of Statistics

Iowa State University

Ames, Iowa 50011 USA

\section{REFERENCES}

Cressie, N., 1988, Spatial Prediction and Ordinary Kriging: Math. Geol., v. 20, p. 405-421.

Rivoirard, J., 1987. Two Key Parameters When Choosing the Kriging Neighborhood: Math. Geol., v. 19, p. $851-856$. 